\title{
CARACTERIZAÇÃO QUÍMICA DE SOLO CONTAMINADO POR RESÍDUOS SÓLIDOS URBANOS NA ESTRADA ARROIO PAVUNA EM JACAREPAGUÁ NO MUNICÍPIO RIO DE JANEIRO
}

Adriana Alves Barbosa ${ }^{1}$ Sérgio Machado Corrêa ${ }^{2}$

Resumo: Este trabalho avaliou o grau de contaminação de um solo em contato com resíduos sólidos lançados diariamente pelos moradores da comunidade Arroio Pavuna, em Jacarepaguá (RJ). Por causa da presença de urubus na região estudada, o local é também conhecido por estrada do urubu. Para uma caracterização química, foram determinados $\mathrm{pH}$, Carbono Orgânico, Matéria Orgânica, alguns metais como Ni, Cu, Fe, Cd, $\mathrm{Pb}, \mathrm{Zn}, \mathrm{Cr}$, além disso foi realizado um teste toxicológico com oligoquetas Eisenia andrei para avaliar a função de habitat deste solo. Antes de prosseguir o estudo, os métodos foram validados. Os resultados obtidos apontaram que a região apresenta um solo ácido na faixa de $\mathrm{pH}$ de 5,51 e 6,70, com concentrações de $\mathrm{Cu}, \mathrm{Cr}$, $\mathrm{Ni}$ e $\mathrm{Zn}$ acima dos valores orientadores de referência de qualidade dos solos, bem como um teor médio de matéria orgânica e também este solo apresentou função de habitat limitada.

Palavras-Chave: Resíduos sólidos, caracterização química, remediação.

Abstract: This study evaluated the degree of contamination of soil in contact with solid waste launched daily by community residents Arroio Pavuna in Jacarepaguá (RJ). Because of the presence of vultures in the region studied, the place is also known as the vulture road. For the chemical analysis, were determined $\mathrm{pH}$, organic carbon, organic matter, some metals such as $\mathrm{Ni}, \mathrm{Cu}, \mathrm{Fe}, \mathrm{Cd}, \mathrm{Pb}, \mathrm{Zn}, \mathrm{Cr}$ and a toxicology test was performed with earthworms Eisenia andrei to evaluating the function of this soil habitat. Before proceeding to the study, the methods were validated. The results showed that the region presents an acid soil in the $\mathrm{pH}$ range of 5.51 and 6.70, with concentrations of $\mathrm{Cu}, \mathrm{Cr}, \mathrm{Ni}$ and $\mathrm{Zn}$ above the guiding reference values of soil quality, as well as an average grade organic matter, moreover, the soil is limited due habitat.

Keywords: solid waste, chemical characterization, remediation.

\footnotetext{
${ }^{1}$ Faculdade de Tecnologia, Universidade do Estado do Rio de Janeiro. E-mail:adriana.balves10@gmail.com

${ }^{2}$ Faculdade de Tecnologia, Universidade do Estado do Rio de Janeiro. E-mail: sergiomc@uerj.br
} 


\section{INTRODUÇÃO}

Uma das consequências mais agravantes do crescimento desordenado da população mundial pelo parâmetro ambiental é a destinação final dos resíduos e a quantidade de lixo gerada todos os dias por bilhões de pessoas. No Brasil, por exemplo, cada brasileiro participa com 1,2 kg de lixo por dia, na Europa $1,3 \mathrm{~kg}$ por habitante e nos Estados Unidos até $2,8 \mathrm{~kg}$ por pessoa, dados do ano de 2010 da Associação Brasileira de Empresas de Limpeza Pública e Resíduos Especiais (ABRELPRE, 2010). Em 2012, o Programa das Nações Unidas para o Meio Ambiente (PNUMA) divulgou que a quantidade de lixo mundial aumentará de 1,3 bilhão de toneladas para 2,2 bilhões de toneladas até o ano de 2025.

Resíduos sólidos, popularmente conhecidos como lixo, são definidos pela Associação Brasileira de Normas Técnicas (ABNT), na norma 10.004 de 2004, como resíduos nos estados sólido e semi-sólido, resultantes de atividades de origem industrial, doméstica, hospitalar, comercial, agrícola, de serviços e de varrição. Ficam incluídos nesta definição os lodos provenientes de sistema de tratamento de água, aqueles gerados em equipamentos e instalações de controle de poluição, bem como determinados líquidos, cujas particularidades tornem inviáveis o seu lançamento na rede pública de esgotos ou corpos de água, ou exijam para isso soluções técnicas e economicamente viáveis em face à melhor tecnologia disponível.

Esses resíduos são classificados em Classe I ou resíduos perigosos, por apresentarem periculosidade, em função de suas propriedades físicas, químicas e infecto contagiosas, acarretando riscos à saúde pública e ao meio ambiente, e em Classe II ou resíduos não perigosos, por não se enquadrar na definição anterior. Sendo os resíduos de Classe II subdivididos em A, resíduos não inertes e em $\mathrm{B}$, resíduos inertes (ABNT, 2004).

De acordo com um estudo de caracterização dos resíduos sólidos urbanos (RSU), isto é, resíduos gerados por atividades domésticas, apresentado pelo Instituto Brasileiro de Geografia e Estatística (IBGE) em 2010, foram verificados que $31,9 \%$ em massa da composição dos RSU são de materiais recicláveis, enquanto que $51,4 \%$ em massa são de matéria orgânica, ou seja, restos de alimentos.

Entretanto, os resíduos apresentam diversas substâncias perigosas, tais como produtos de limpeza, em destaque a água sanitária, os dedetizantes, os vários tipos de plásticos, as embalagens metálicas, as lâmpadas, as pilhas entre outros produtos que são descartados juntos, pois é compreendido lixo como o que é sujo, ou o que sobra e o que não tem mais valor.

Existem diversas formas de destinação dos resíduos sólidos como os aterros sanitários e controlados, os lixões, a compostagem, a reciclagem, a incineração, entre outras. Porém, no Brasil, os aterros são as principais vias de destinação final dos RSU. Sabe-se também que existem muitas rotas clandestinas e irregulares de disposição desses resíduos, que são os lixões. Algumas metas como eliminação dos aterros controlados e lixões até 2014, assim como a implantação da logística reversa pós-consumo de embalagens em geral até 2020, bem como a divulgação de conceitos e a prática da sustentabilidade nos setores industriais e na sociedade civil foram propostas pelo recente Plano Nacional de Resíduos Sólidos (PNRS) de 2011.

Inúmeros são os exemplos de catástrofes ocasionadas pela ausência de um monitoramento ambiental em áreas destinadas inadequadamente à disposição de resíduos sólidos. Acontecimentos como a Cidade dos Meninos em Duque de Caxias (RJ), onde uma fábrica que sintetizava o pesticida hexaclorociclohexano $(\mathrm{HCH})$ foi desativada em 1960, deixando os resíduos deste pesticida expostos no solo até 1989 . Ou como em 2010, quando ocorreu o desmoronamento do morro do Bumba, um antigo lixão em Niterói (RJ). Ou ainda como em 2011, o risco de explosão de um shopping construído sobre um antigo lixão em São Paulo.

Por fim, este trabalho relaciona os problemas ambiental e social pelo conhecimento químico, apresentando um cenário bastante comum na região de Curicica em Jacarepaguá (RJ), ou seja, 
presença de moradias desordenadas próximas aos rios assoreados por lixo e esgoto doméstico, bem como a disposição inadequada de resíduos no solo.

\section{MATERIAIS E MÉTODOS}

\section{1. ÁREA DE ESTUDO}

A região escolhida caracterização química do para contaminado por resíduos sólidos urbanos é a estrada Canal Arroio Pavuna, situada em Jacarepaguá, na zona oeste do município Rio de Janeiro. Esta região é também conhecida como "Estrada do Urubu", porque esses animais rondam, atraídos pelos resíduos. Essa área é permeada pelo rio Arroio Pavuna que já se encontra assoreado, principalmente pela elevada carga de esgoto lançada diariamente pelas moradias à beira do rio e pelas tubulações clandestinas. Além disso, esse é um dos principais rios que deságua na Lagoa de Jacarepaguá, hoje totalmente contaminada.

O estudo do solo desta região ocorreu em dois períodos de fevereiro de 2013 (verão) e junho de 2013 (inverno) e envolveu quatro fases. A primeira fase foi realizada com coletas de amostra do solo contaminado. No segundo momento, ocorreram as análises físico-químicas, um teste biológico e as análises instrumentais.

Foram escolhidos sete pontos representativos de solo contaminado por resíduos sólidos. A Figura 1 apresenta na forma de zig zag as coletas das amostras de solo.

As amostras dos pontos 1 e 2 representavam amostras bastante impactadas por resíduos domésticos e de materiais de construção. As amostras do ponto 3 eram contaminadas por fezes de animais. Já as amostras dos pontos 4 e 7 estavam longe de moradias, com pequenas concentrações de lixo enquanto as amostras dos pontos 5 e 6 foram coletadas próximas às saídas clandestinas de esgoto doméstico.

As metodologias utilizadas para as análises pertencem a órgãos de credibilidade no campo científico como a Empresa Brasileira de Pesquisa Agropecuária (EMBRAPA), a Companhia de Tecnologia e Saneamento Ambiental do Estado de São Paulo (CETESB) e a Agência de Proteção Ambiental dos Estados Unidos (USEPA). Além disso, esses métodos foram validados, a fim de obter resultados adequados e confiáveis à qualidade pretendida de acordo com os parâmetros do Instituto Nacional de Metrologia (INMETRO).

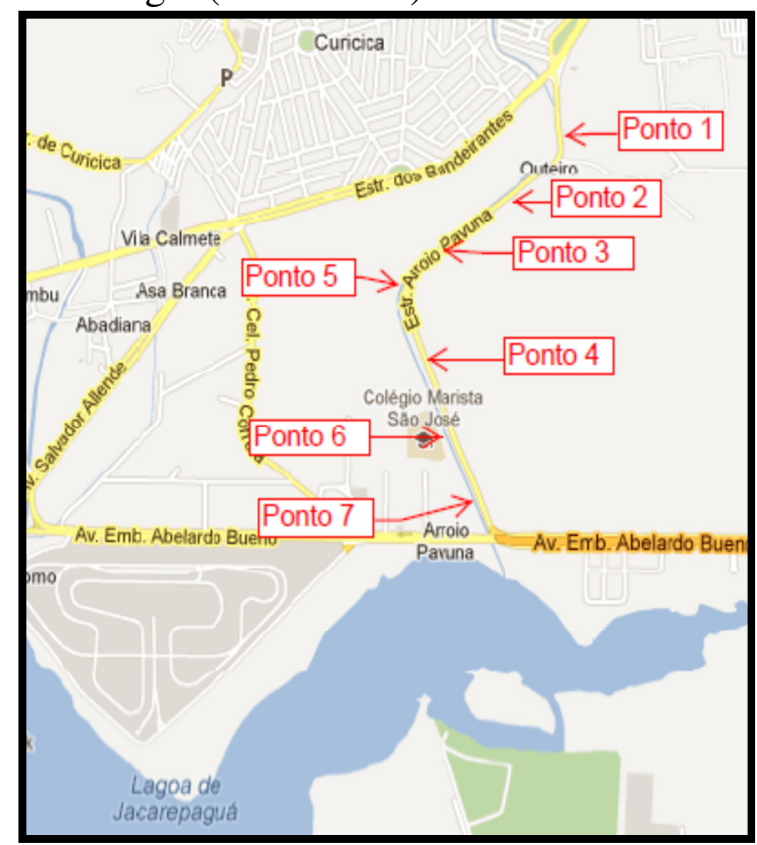

Figura 1. Localização da região em estudo, com os sete pontos de coleta.

A coleta das amostras foi dirigida pelas metodologias de análise e amostragem de solos da EMBRAPA (2011) e CETESB (1999). Inicialmente, a região foi dividida de maneira uniforme e a distribuição dos pontos de amostragem foi direcionada de acordo com as fontes ou vias de contaminação. Com o auxílio de uma pá, as amostras foram coletadas nos 7 pontos, a uma profundidade média de $20 \mathrm{~cm}$, tendo o cuidado de limpar a superfície da área escolhida, removendo folhas, galhos, outros materiais interferentes. Cerca de $500 \mathrm{~g}$ de solo de cada ponto foram armazenados em sacos de polietileno lacrados e identificados.

Depois, as amostras foram secas ao ar livre numa temperatura inferior a $40^{\circ} \mathrm{C}$.

$\mathrm{O}$ tempo de secagem variou com a umidade da amostra. Em seguida, a amostra foi moída com o auxílio de almofariz e pistilo de porcelana (não foram moídos cascalhos e calhaus). Com isso, o material foi acondicionado em um recipiente adequado, podendo ser um saco plástico ou 
um frasco de vidro, dependendo da análise que será realizada (Galvani et al., 2005).

Foram realizadas algumas análises físicas e químicas, como $\mathrm{pH}$ e carbono orgânico, seguindo o manual de métodos de análises de solos da EMBRAPA (2011). Posteriormente, a determinação da concentração dos metais Alumínio, Cádmio, Chumbo, Cobre, Ferro, Níquel e Zinco foi realizada conforme a metodologia U.S. EPA 3050B e a análise química em um equipamento Perkin Elmer modelo AA400.

\subsection{CARBONO ORGÂNICO}

Este teste se baseia no processo de titulação, com mudança de coloração azul para verde. Inicialmente, a amostra é diluída na solução de dicromato de potássio $0,0667 \mathrm{~mol} \mathrm{~L}^{-1}$. Em seguida, a amostra é titulada com a solução de sulfato ferroso amoniacal $0,1 \mathrm{~mol} \mathrm{~L}^{-1}$, na presença do indicador difenilamina. Determina-se a quantidade de carbono orgânico por meio das Equações 1, 2 e 3.

$$
\begin{aligned}
& \mathrm{C}^{1}=\left(40-\mathrm{V}^{2}\right) \times \mathrm{F}^{3} \times 0,6 \\
& \mathrm{~F}^{3}=\left(40 / \mathrm{V}^{4}\right) \\
& \mathrm{MO}^{5}=\mathrm{C}^{1} \times 1,724
\end{aligned}
$$

$\mathrm{C}^{1}$ :Carbono Orgânico $\left(\mathrm{g} \mathrm{kg}^{-1}\right)$

$\mathrm{V}^{2}$ :Volume gasto

$\mathrm{F}^{3}$ : Fator de diluição

$\mathrm{V}^{4}$ : Volume gasto no branco

$\mathrm{MO}^{5}$ : Matéria Orgânica $\left(\mathrm{g} \mathrm{kg}^{-1}\right)$

\subsection{METODOLOGIA DE ESPECTROMETRIA DE ABSORÇÃO ATÔMICA POR CHAMA}

As espécies metálicas escolhidas para a análise do solo em estudo foram Alumínio, Cádmio, Chumbo, Cobre, Cromo, Ferro, Níquel e Zinco. Os valores utilizados como referência na avaliação deste solo são aqueles estabelecidos na Resolução Conama $n^{\circ} 420$ de 2009. O procedimento desta metodologia é baseado na digestão das amostras de solo, com adições repetidas de ácido nítrico e peróxido de hidrogênio, seguido de aquecimento e refluxo até que uma coloração marrom apareça no condensador, significando a oxidação da amostra. Por fim, cada amostra é reduzida a $5 \mathrm{~mL}$, filtrada e posteriormente analisada no equipamento Perkin Elmer modelo AA400.

\subsection{TESTE DE COMPORTAMENTO}

Realizou-se o teste de comportamento de fuga com oligoquetas da espécie Eisenia Andrei, a fim de conhecer a função de habitat do solo em estudo (ISO, 2002). O teste ocorreu em triplicata. Inicialmente, as oligoquetas foram condicionadas em solo artificial (composto por $70 \%$ de areia, 20\% de caulim, $10 \%$ de pó de casca de coco) por 24 horas. No segundo momento, foram colocados 600 gramas de solo artificial e a mesma quantidade de solo contaminado em um recipiente de plástico, com auxílio de um divisor. Posteriormente, foram introduzidas as 10 oligoquetas no recipiente. $\mathrm{O}$ teste ocorreu por 48 horas, a temperatura de $22^{\circ} \mathrm{C}$, com ciclos de luz e escuridão de 12 horas. No final do teste, calculou-se, em percentagem, a quantidade de espécie presente. Tanto as oligoquetas quanto os componentes do solo artificial foram doados pelo Centro de Tecnologia Mineral (CETEM).

\section{RESULTADOS E DISCUSSÃO}

Em geral, os solos brasileiros são ácidos devido ao intenso intemperismo tropical das rochas, à presença de ácidos orgânicos e inorgânicos, à decomposição dos materiais orgânicos e aos elevadores teores dos íons $\mathrm{Al}^{+3}$ em solução no solo. Os sete pontos do solo analisado apresentaram pH moderadamente ácido.

Os valores de matéria orgânica são classificados em teor alto quando maior que $45 \mathrm{~g} \mathrm{~kg}^{-1}$, em teor médio na faixa de $15 \mathrm{a}$ $45 \mathrm{~g} \mathrm{~kg}^{-1}$ e teor baixo aqueles menores que $15 \mathrm{~g} \mathrm{~kg}^{-1}$. De acordo com essa classificação e com os valores apresentados nas Tabelas 1 e 2, os sete pontos do solo analisado apontaram um teor médio de matéria orgânica.

As concentrações das substâncias inorgânicas ou orgânicas são parâmetros para classificar o grau de contaminação de um solo. Os metais escolhidos foram Al, $\mathrm{Cd}, \mathrm{Pb}, \mathrm{Cu}, \mathrm{Cr}, \mathrm{Fe}$, Ni e $\mathrm{Zn}$, apresentados separadamente nas Figuras 2 a 8. 


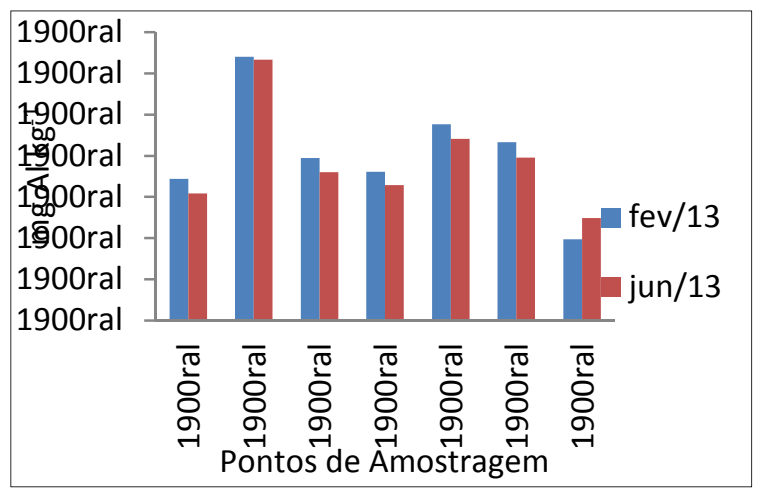

Figura 2. Teor de Al nas sete amostras de solo.

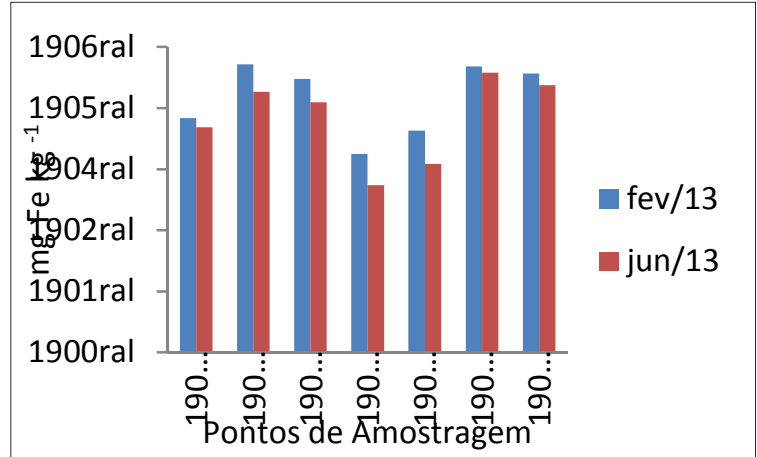

Figura 3. Teor de Ferro nas sete amostras de solo.

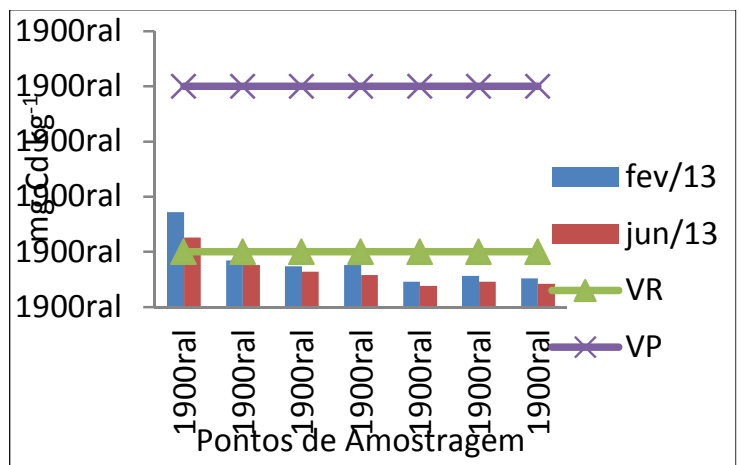

Figura 4. Teor de Cádmio nas sete amostras de solo.

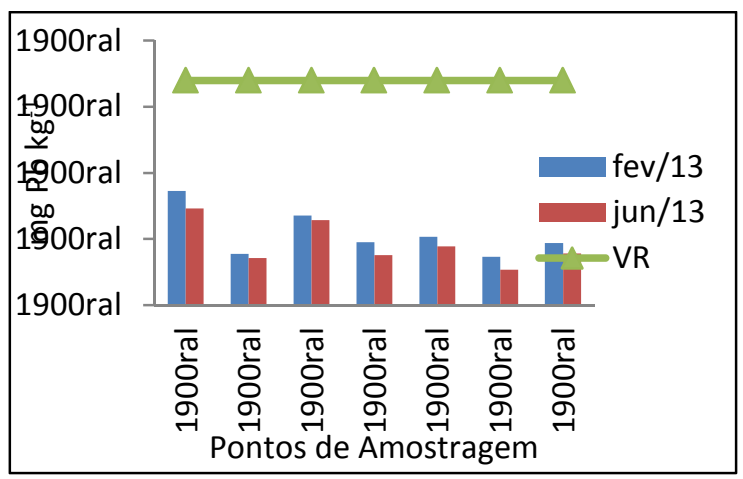

Figura 5. Teor de Chumbo nas sete amostras de solo.

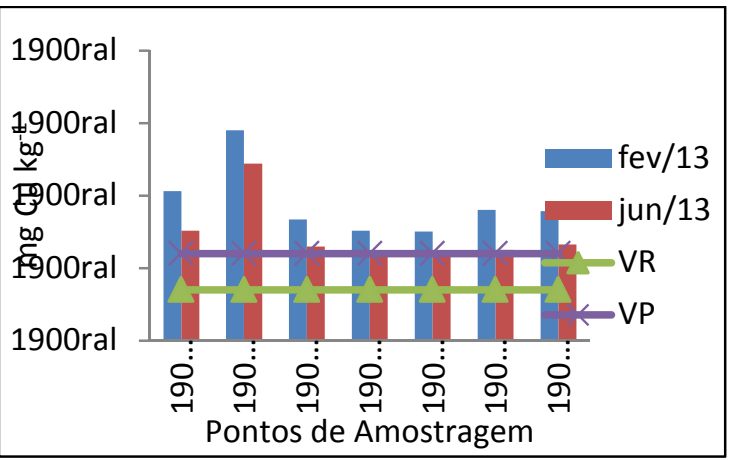

Figura 6. Teor de Cobre nas sete amostras de solo.

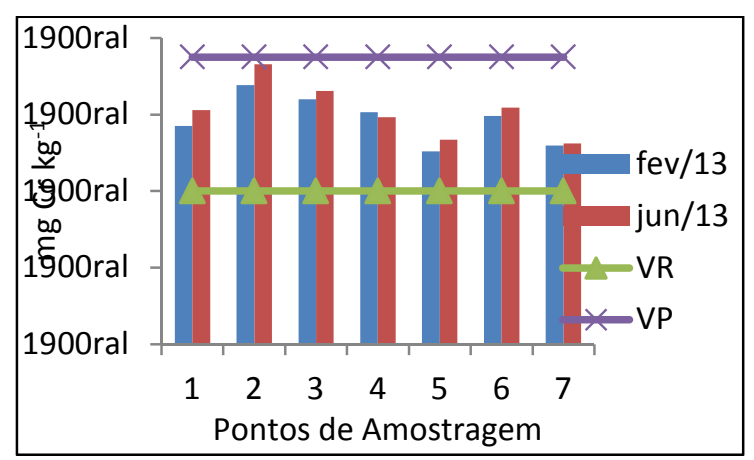

Figura 7. Teor de Cromo nas sete amostras de solo.

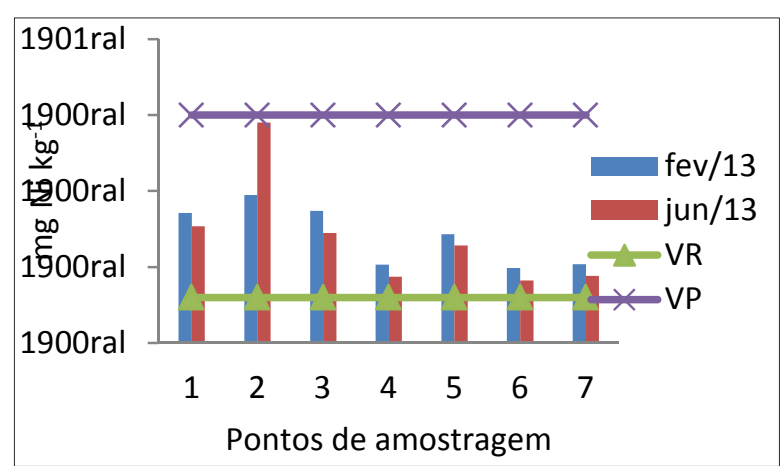

Figura 7. Teor de Níquel nas sete amostras de solo.

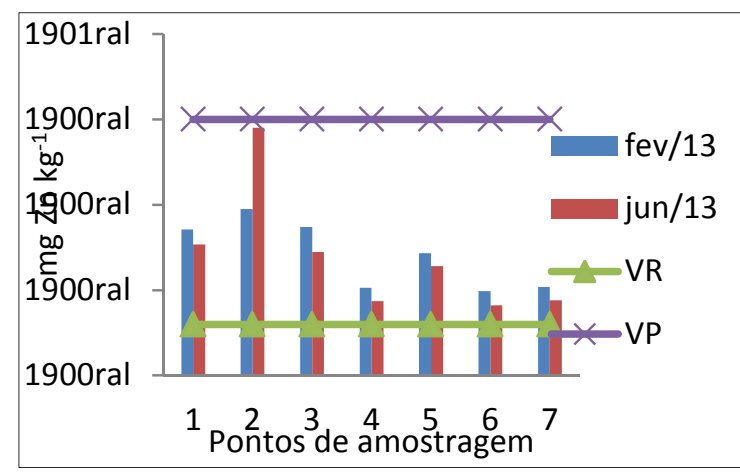

Figura 8. Teor de Zinco nas sete amostras de solo.

Dentre os oito metais analisados, apenas Fe e Al não apresentam Valores de Referência de Qualidade (VRQ), Valores de 
Prevenção (VP) e Valores de Intervenção Residencial (VIR), pois não é possível constatar se a concentração é resultante de vias de contaminação ou do material de origem da rocha matriz.

Os metais $\mathrm{Cd}$ (com exceção do ponto 1) e $\mathrm{Pb}$ apresentaram valores de concentração abaixo do limite dos valores de referência de qualidade do solo. O elemento $\mathrm{Zn}$ apresentou valores de concentração acima de VRQ, porém abaixo de VP, já a concentração de $\mathrm{Cu}$ foi acima do VP. O Ni apresentou alguns pontos acima de VRQ. O Cr apresentou valores acima de VRQ.

$\mathrm{O}$ ponto 2 na análise apresentou valores elevados de concentração em grande parte dos metais, evidenciando a contribuição do lixo doméstico, principalmente o lixo eletrônico como pilhas, baterias, eletrodomésticos descartados de maneira inadequada, conforme pode ser visualizado na Figura 9.

O teste de comportamento com oligoquetas representado na Figura 10 confirmou que o solo analisado apresentou função de habitat limitada, tendo em vista que menos de $20 \%$ dos organismos foram encontrados no solo contaminado por resíduos sólidos nos dois períodos de análises.
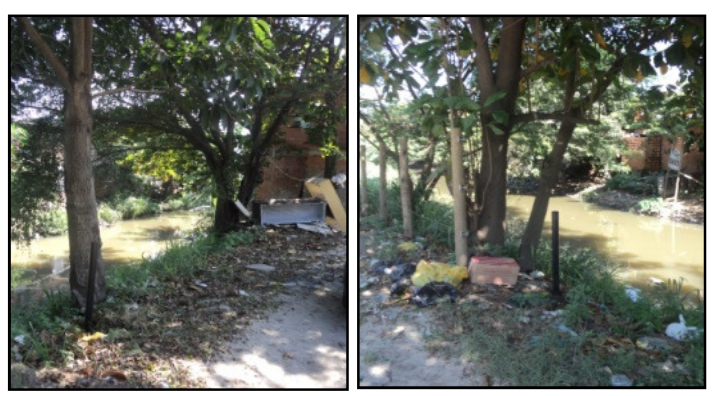

Figura 9. Imagens do descarte de resíduos domésticos na região de estudo deste trabalho.

Tabela 1. Propriedades físico-químicas dos pontos analisados do solo em fevereiro de $2013 \mathrm{em} \mathrm{g} \mathrm{kg}^{-1}$

\begin{tabular}{cccc}
\hline Ponto & $\mathrm{pH}$ & $\begin{array}{c}\text { Carbono } \\
\text { Orgânico }\end{array}$ & $\begin{array}{c}\text { Matéria } \\
\text { Orgânica }\end{array}$ \\
1 & 6,09 & 16,80 & 29,23 \\
2 & 6,18 & 14,40 & 24,82 \\
3 & 6,44 & 15,36 & 26,48 \\
4 & 6,70 & 12,48 & 21,71 \\
5 & 5,96 & 17,28 & 29,79 \\
6 & 5,82 & 15,36 & 26,48 \\
7 & 5,51 & 16,32 & 28,13 \\
\hline
\end{tabular}

Tabela 2. Propriedades físico-químicas dos pontos analisados do solo em junho de $2013 \mathrm{em} \mathrm{g} \mathrm{kg}^{-1}$

\begin{tabular}{cccc}
\hline Ponto & $\mathrm{pH}$ & $\begin{array}{c}\text { Carbono } \\
\text { Orgânico }\end{array}$ & $\begin{array}{c}\text { Matéria } \\
\text { Orgânica }\end{array}$ \\
\hline 1 & 6,05 & 15,96 & 27,51 \\
2 & 6,15 & 14,69 & 25,32 \\
3 & 6,31 & 15,74 & 27,13 \\
4 & 6,23 & 12,18 & 20,99 \\
\hline 5 & 6,26 & 16,61 & 28,63 \\
6 & 5,78 & 16,28 & 28,06 \\
7 & 6,40 & 15,23 & 26,25 \\
\hline
\end{tabular}

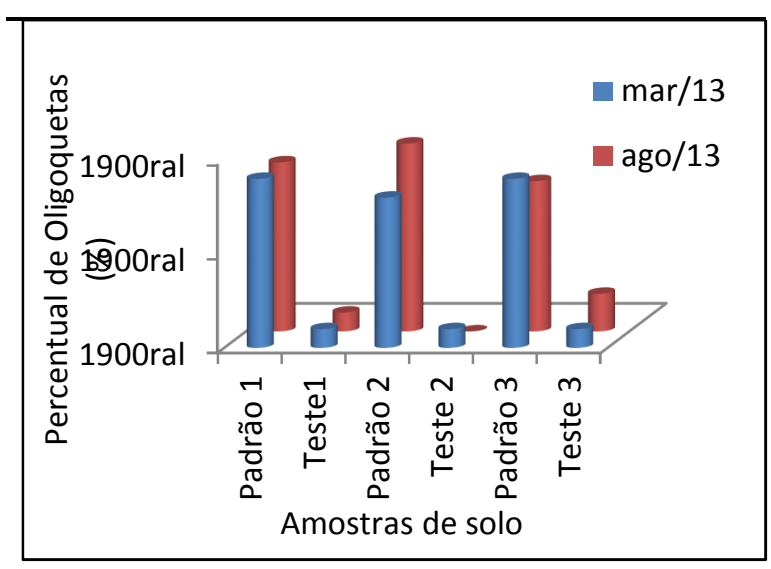

Figura 10. Teste com oligoquetas.

\section{CONCLUSÕES}

$\mathrm{O}$ solo em estudo apresentou $\mathrm{pH}$ moderadamente ácido nos dois períodos das análises, o que contribui para a solubilização das espécies metálicas e dos compostos orgânicos existentes na região. Além disso, a acidez de um solo pode afetar o habitat de algumas espécies. De acordo com o teste de comportamento com oligoquetas, verificou-se que o solo contaminado por lixo interferiu na sobrevivência destes organismos.

As amostras de solo revelaram quatro metais com valores de concentração acima dos valores de referência de qualidade estabelecidos pela Resolução Conama $\mathrm{n}^{\mathrm{o}}$ 420 de 2009. E, por apresentar pelo menos um metal com concentração maior que o valor de prevenção e menor ou igual ao valor de intervenção, este solo é classificado quanto à sua qualidade em classe três. Como procedimento de controle e prevenção da qualidade deste solo, a legislação requer a identificação da fonte potencial da contaminação, avaliação da ocorrência natural da substância química, assim como o controle das fontes de 
contaminação e monitoramento da qualidade do solo e da água subterrânea.

Faz-se necessário a continuação do monitoramento ambiental para se escolher uma técnica de remediação da região pelos aspectos físicos, químicos ou biológicos.

Além disso, esse trabalho alerta para a disposição inadequada de quaisquer resíduos no solo, pois dependendo do tempo de exposição, a região pode conter concentrações elevadas de substâncias aparentemente não tóxicas e afetar a qualidade do ambiente local, assim como a saúde da população residente na região.

\section{REFERÊNCIAS}

ABNT - Agência Brasileira de Normas Técnicas - Norma NBR 6502. 1995. Tamanho de partículas de solos, Rio de Janeiro.

ABRELPE - Associação Brasileira de Empresas de Limpeza Pública e Resíduos Especiais. Disponível em: <http://www.abrelpe.org.br/> Acesso em: 23 mar. 2013.

CESAR, R.G.; EGLER, S.G.; ALAMINO, R.C.J.; POLIVANOV, H.; SILVA, R.C CASTILHOS, Z.C. \& ARAUJO, P.C. 2008. Avaliação do potencial tóxico de latossolos e chernossolos acrescido de lodo de esgoto utilizando bioensaios com oligoquetas da espécie Eisenia andrei. Rio de Janeiro: Anuário do Instituto de Geociências da UFRJ, 31(2), 53-60.

CETEM. 2006. Centro de Tecnologia Mineral. Biorremediação de solos contaminados por petróleo: ênfase no uso de biorreatores. Rio de Janeiro: Série Tecnologia Ambiental.

CETESB - Centro de Tecnologia e Saneamento Básico do Estado de São Paulo.2013.<http://www.cetesb.sp.gov.br/te cnologia-ambiental/laboratorios/109informacoes-toxicologicas> Acesso em: 13 mai. 2013

CONAMA-Conselho Nacional do Meio Ambiente. Resolução $n^{\circ} 420$ de 28 de dezembro de 2009: critérios e valores orientadores de qualidade do solo quanto à presença de substâncias químicas, ed.
Diário Oficial da União $\mathrm{n}^{0} 249$, de 30 dez.2009;81-84.

COSTA, W.; MATIAS, N. D. 2013. Estudo químico de alguns pontos do solo superficial do aterro controlado do Botuquara. Paraná: Ambiência Guarapuava, 8(1), 85-99.

EMBRAPA- Empresa Brasileira de Pesquisa Agropecuária.2011. Centro Nacional de Pesquisa de Solos. In: Manual de Métodos de Análises de Solo. $2^{\mathrm{a}}$ ed. Rio de Janeiro, $88 \mathrm{p}$.

GALVANI, F. \& FERNANDES, F.A. 2005. Registro e preparação de amostra de solos para análises físicas e químicas. Corumbá, MS.

INMETRO, Instituto Nacional de Metrologia. Orientação sobre validação de métodos de ensaios químicos, 2007.

MMA - Ministério do Meio Ambiente. Plano Nacional de Resíduos Sólidos. Brasília, 2011,102p.

PREFEITURA DO RIO, 2012. Disponível em: <http://www.rio.rj.gov.br/web/guest>. Acesso em: 9 out. 2012.

ROCCO, Rogério. 2012. "História da Legislação Ambiental Brasileira: um passeio pela legislação, pelo direito ambiental e por assuntos correlatos" in AHMED, Flávio e COUTINHO, Ronaldo. Curso de Direito Ambiental. Rio de Janeiro: Lumen Juris/OAB-RJ.

SILVA, C.; SCHOENHALS, M. \& ARANTES, E.J. 2008. Diagnóstico da contaminação do solo na área de disposição de resíduos sólidos de PeabirúPR.

SUZUKI, E.Y.; TAIOLI,F. \& RODRIGUES, C.L. 2005. Avaliação do comportamento do solo da região do lixão de Ilhabela - SP. Águas Subterrâneas, 19, 67-76.

YEUNG, A.T. \& GU, Y.Y. 2011. A review on techniques to enhance electrochemical remediation of contaminated soils. Journal of Hazardous Materials, 195, 11-29. 